\title{
The impact of radiotherapy on clinical outcomes in parameningeal rhabdomyosarcoma
}

\author{
Yunseon Choi, MD, PhD ${ }^{1,2}$, Do Hoon Lim, MD, PhD \\ 'Department of Radiation Oncology, Samsung Medical Center, Sungkyunkwan University School of Medicine, Seoul; \\ ${ }^{2}$ Department of Radiation Oncology, Inje University Busan Paik Hospital, Inje University College of Medicine, Busan, Korea
}

Purpose: Radiotherapy (RT) is considered a mainstay of treatment in parameningeal rhabdomyosarcoma (PM-RMS). We aim to determine the treatment outcomes and prognostic factors for PM-RMS patients who treated with RT. In addition, we tried to evaluate the adequate dose and timing of RT.

Materials and Methods: Twenty-two patients with PM-RMS from 1995 to 2013 were evaluated. Seven patients had intracranial extension (ICE) and 17 patients had skull base bony erosion (SBBE). Five patients showed distant metastases at the time of diagnosis. All patients underwent chemotherapy and RT. The median radiation dose was $50.4 \mathrm{~Gy}$ (range, 40.0 to $56.0 \mathrm{~Gy}$ ).

Results: The median follow-up was 28.7 months. Twelve patients (54.5\%) experienced failure after treatment; 4 local, 2 regional, and 6 distant failures. The 5-year local control (LC) and overall survival (OS) were $77.7 \%$ and 38.5\%, respectively. The 5-year OS rate was $50.8 \%$ for patients without distant metastases and $0 \%$ for patients with metastases ( $p<0.001)$. Radiation dose (<50 Gy vs. $\geq 50 \mathrm{~Gy})$ did not compromise the LC ( $p=0.645)$. However, LC was affected by ICE ( $p=0.031)$. Delayed administration ( $>22$ weeks) of RT was related to a higher rate of local failure (40.0\%).

Conclusion: RT resulted in a higher rate of local control in PM-RMS. However, it was not extended to survival outcome. A more effective treatment for PM-RMS is warranted.

Key words: Meninges, Rhabdomyosarcoma, Radiotherapy, Local control

\section{Introduction}

Rhabdomyosarcoma (RMS) mainly occurs in the head and neck area, and the parameningeal site accounts for 15\%-20\% of all RMS [1]. According to the Intergroup RMS Study III [2], parameningeal rhabdomyosarcoma (PM-RMS) constitutes 41\% of head and neck-based RMS.

PM-RMS refers to tumors occurring in the nasal cavity, paranasal sinuses, infratemporal fossa, pterygoid palatine fossa, nasopharynx, and the mastoid or middle ear. The PM-
RMS, originating in the skull base, is invasive, and sometimes it can develop into neoplastic meningitis [1]. Hence, the parameningeal site has a poor prognosis compared with other locations of RMS $[1,3]$.

The current treatment guidelines for RMS emphasize the importance of a multimodal approach [4]. However, surgical resection with an adequate margin is difficult because of its deep location, which is close to several important structures, such as the brain, cranial nerve, and many vessels. Therefore, radiotherapy (RT) with chemotherapy is considered a mainstay

Received 10 May 2016, Revised 7 July 2016, Accepted 22 July 2016.

Correspondence: Do Hoon Lim, MD, PhD, Department of Radiation Oncology, Samsung Medical Center, Sungkyunkwan University School of Medicine, 81, Irwon-ro, Gangnam-gu, Seoul 06351, Korea. Tel: +82-2-3410-2600, Fax: +82-2-3410-2619, E-mail: dh8lim@skku.edu

(c) This is an Open Access article distributed under the terms of the Creative Commons Attribution Non-Commercial License (http://creativecommons.org/ licenses/by-nc/4.0/) which permits unrestricted non-commercial use, distribution, and reproduction in any medium, provided the original work is properly cited.

www.e-roj.org 
of treatment in PM-RMS.

In PM-RMS, the location of the tumor is known to be correlated with the prognosis. In particular, PM-RMS in the paranasal sinus, infratemporal fossa, or pterygopalatine fossa showed an unfavorable outcome according to a recent report by Merks et al. [5]. In addition, alveolar histology was shown to be related to poor prognosis, compared with embryonal histology according to a report by Turner and Richmon [6].

Early initiation of RT with chemotherapy is generally recommended if there is a possibility of meningeal (intracranial) involvement in cases with skull base bony erosion (SBBE), cranial nerve palsy (CNP), and intracranial extension (ICE) [7]. In particular, ICE is considered to the most important adverse prognostic factor [1] and the early application of RT (0-4 weeks) is often considered in ICE or CNP cases. However, the recommended optimal timing for $\mathrm{RT}$ is still variable, according to clinical protocols [7-9].

The current guideline for RT is a dosage of $1.8 \mathrm{~Gy} /$ day, up to a total of $50.4 \mathrm{~Gy}$, using a $1.5-2 \mathrm{~cm}$ margin around the tumor [4]. Michalski et al. [10] argued that a dose of at least $47.5 \mathrm{~Gy}$ was associated with lower rates of local failure. In contrast, Puri et al. [11] reported that a comparable local control (2-year local control [LC], 84\%) was achieved with a reduced dose of 36 Gy external beam RT in very young patients ( $\leq 36$ months). The Intergroup Rhabdomyosarcoma Study $V$ also used a reduced dose radiation (36.0-50.4 Gy) in very young patients. In this point of view, a radiation dose reduction can be considered in patients with young age or a low-risk of relapse [12].

Studies about the treatment of PM-RMS are rare and difficult because of the low incidence of this disease [13]. Thus, most studies about PM-RMS have been designed to use retrospectively collected data.

This study aimed to investigate RT outcomes and its clinically related prognostic factors. Delaying $R T$ or inadequate $R T$ for those with high risk features may reduce survival and LC. Therefore, we additionally evaluated an adequate dose and timing of the RT.

\section{Materials and Methods}

\section{Patients}

In this study 22 patients were identified and evaluated with PM-RMS and received RT and chemotherapy at the Samsung Medical Center in Seoul, Korea from May 1995 to April 2013. A retrospective analysis was performed to assess treatment outcome and prognostic factors for these PMRMS patients. This study was approved by the Samsung
Medical Center Institutional Review Board. The preoperative staging of RMS was defined as follows: stage I, favorable site; stage II, unfavorable site $(<5 \mathrm{~cm})$, NO; stage III, unfavorable site, $(>5 \mathrm{~cm})$ or $\mathrm{N} 1$; stage IV, M1. Every PM-RMS was classified as an unfavorable site according to the criteria. Seventeen patients (77.4\%) underwent MRI and the other 5 patients (22.7\%) underwent CT for diagnosis of meningeal involvement.

\section{Treatment}

For the sequence of treatment, surgical resection or biopsy was initially performed. Chemotherapy followed by RT was administered subsequently.

According to the Intergroup Rhabdomyosarcoma Study (IRS)-III protocol, the chemotherapy regimen was composed of vincristine, adriamycin (or actinomycin D), cyclophosphamide and cis-platinum (or cis-platinum plus etoposide). The IRSIV chemotherapy protocol includes vincristine, dactinomycin and cyclophosphamide (the most common); or vincristine, dactinomycin, and ifosfamide; or vincristine, ifosfamide, and etoposide.

For RT planning, the patients were fixed with a thermoplastic mask during a simulation computed tomography (CT) scanning. The CT images were used in all patients for treatment planning. For RT, a three-dimensional conformal radiotherapy technique was commonly used. However, an intensity-modulated RT was also used, if deemed necessary by the physicians. A total of 50.4 Gy was generally given to control the tumor. However, young patients $(<10$ years, $n=2)$, or patients with a favorable response to initial chemotherapy, were considered for a reduced dose $\mathrm{RT}$ (<50 Gy). The clinical target volume (CTV) was defined as $1.0-1.5 \mathrm{~cm}$ margin to gross tumor volume. The planning target volume was defined as an additional $0.5 \mathrm{~cm}$ margin to CTV. Brainstem, cochleas, optic nerves, optic chiasm, and lens were drawn as organs at risk.

\section{Statistical analysis}

Events were categorized according to local, regional (lymph nodal), and distant failure. Failure was defined as an increased size of a pre-existing tumor or newly developed disease at any site.

A Fisher's exact test was used to find the clinical factors related to treatment outcomes. The Kaplan-Meier method and log-rank test were used for LC and survival analysis. $\mathrm{LC}$ and survival were defined as time from the diagnosis to local failure and death, respectively. Progression-free survival (PFS) was defined as time from the diagnosis to event (local, 


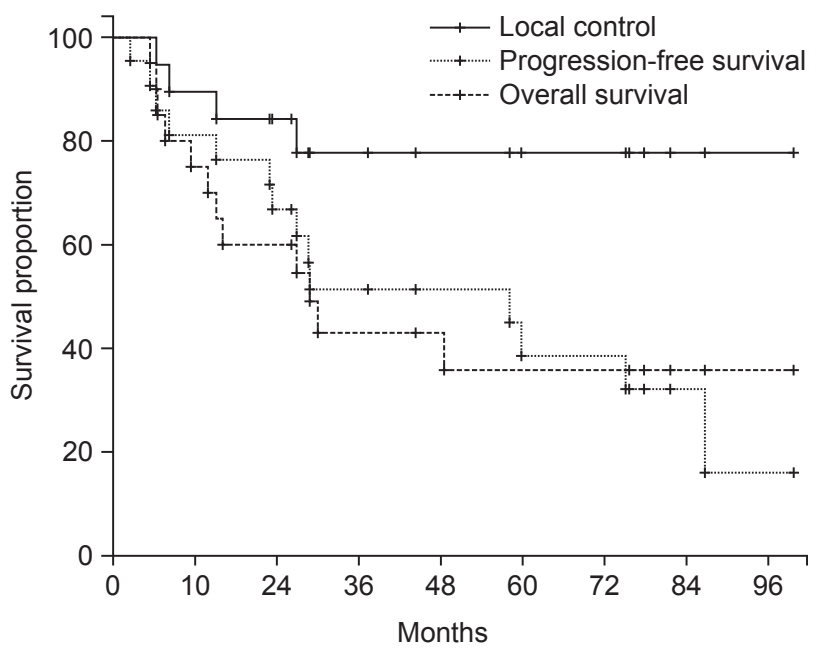

Fig. 1. Local control, progression-free survival, and overall survival.

regional, and distant failure or death).

\section{Results}

\section{Patient and treatment characteristics}

Table 1 summarizes the patient and treatment characteristics. The patient ages ranged from 5 to 60 years old, with a median of 19.5 years, and 5 patients $(22.7 \%)$ were under the age of 10 years. Five patients $(22.7 \%)$ had metastatic disease at diagnosis. High-risk features were as follows; 7 (31.8\%) had ICE, 17 (77.3\%) had SBBE, and 1 (4.5\%) had CNP.

Surgical resection was performed before the RT in 4 patients (18.2\%). Among the 4 patients who underwent surgery before RT, 2 patients underwent gross total resection and other 2 patients underwent partial resection (endoscopic sinus surgery). Among the 4 patients who underwent surgery, 2 patients had tumors less than $5 \mathrm{~cm}$ and 2 patients had tumors equal to or greater than $5 \mathrm{~cm}$. All patients underwent chemotherapy and RT. The most commonly used chemotherapy regimen was the IRS-III regimen 35 ( $n=12,54.5 \%)$. The other 4 patients (18.2\%) received IRS-IV regimen based chemotherapy. The median radiation dose was $50.4 \mathrm{~Gy}$ (range, 40.0 to $56.0 \mathrm{~Gy}$ ).

\section{Pattern of failure and local control}

The median follow-up time was 28.7 months. During the follow-up period, local, regional and distant failures occurred in $4(18.2 \%), 2(9.1 \%)$, and $6(27.3 \%)$ patients. Overall, 12 patients (54.5\%) showed disease progression (1 experienced both local failure and distant failure). Alveolar histology and ICE showed a tendency for higher local failure $(p=0.098$ and
Table 1. Patient characteristics and treatments

\begin{tabular}{|c|c|}
\hline Characteristic & No. $(\%)$ \\
\hline \multicolumn{2}{|l|}{ Sex } \\
\hline Male & 7 (31.8) \\
\hline Female & $15(68.2)$ \\
\hline Age (yr), median (range) & $19.5(3-60)$ \\
\hline \multicolumn{2}{|l|}{ Histology } \\
\hline Embryonal & $9(40.9)$ \\
\hline Alveolar & $9(40.9)$ \\
\hline Pleomorphic & $1(4.5)$ \\
\hline NOS & $3(13.6)$ \\
\hline \multicolumn{2}{|l|}{ Risk factor } \\
\hline Cranial nerve palsy & $1(4.5)$ \\
\hline Skull base bony erosion & $17(77.3)$ \\
\hline Intracranial extension & 7 (31.8) \\
\hline \multicolumn{2}{|l|}{ Site } \\
\hline Nasopharynx & 7 (31.8) \\
\hline Nasal cavity & $6(27.3)$ \\
\hline Paranasal sinus & 7 (31.8) \\
\hline Infratemporal fossa and PPF & $2(9.1)$ \\
\hline \multicolumn{2}{|l|}{ Preoperative stage } \\
\hline Stage II (<5 cm and N0) & $2(9.1)$ \\
\hline Stage III ( $\geq 5 \mathrm{~cm}$ or N1) & $15(68.2)$ \\
\hline Stage IV (M1) & $5(22.7)$ \\
\hline \multicolumn{2}{|l|}{ Treatment method } \\
\hline Surgery & $4(18.2)$ \\
\hline Gross total resection & $2(9.1)$ \\
\hline Partial resection & $2(9.1)$ \\
\hline Chemotherapy & $22(100)$ \\
\hline Radiotherapy & $22(100)$ \\
\hline \multicolumn{2}{|l|}{ RT modality } \\
\hline 3D-CRT & $17(77.3)$ \\
\hline IMRT & $5(22.7)$ \\
\hline \multicolumn{2}{|l|}{ RT field } \\
\hline Primary tumor & 19 (86.4) \\
\hline Primary tumor + regional LN & $3(13.6)$ \\
\hline \multicolumn{2}{|l|}{ RT dose (Gy) } \\
\hline$<50$ & $4(18.2)$ \\
\hline$\geq 50$ & 18 (81.8) \\
\hline
\end{tabular}

NOS, not otherwise specified; PPF, pterygopalatine fossa; RT, radiotherapy; 3D-CRT, three-dimensional conformal radiotherapy; IMRT, intensity-modulated radiotherapy; LN, lymph node.

$p=0.077$, respectively, by Fisher exact test). No secondary malignancy was observed in the follow-up periods.

The 2- and 3-year LC rates were $84.2 \%$ and $77.7 \%$, respectively (Fig. 1).

\section{Survival analysis}

The 2- and 3-year overall survival (OS) rates were $66.8 \%$ and $51.4 \%$, respectively (Fig. 1). The 2- and 3-year PFS rate was $60 \%$ and $43 \%$, respectively. The 5 -year OS rate was $50.8 \%$ 
A

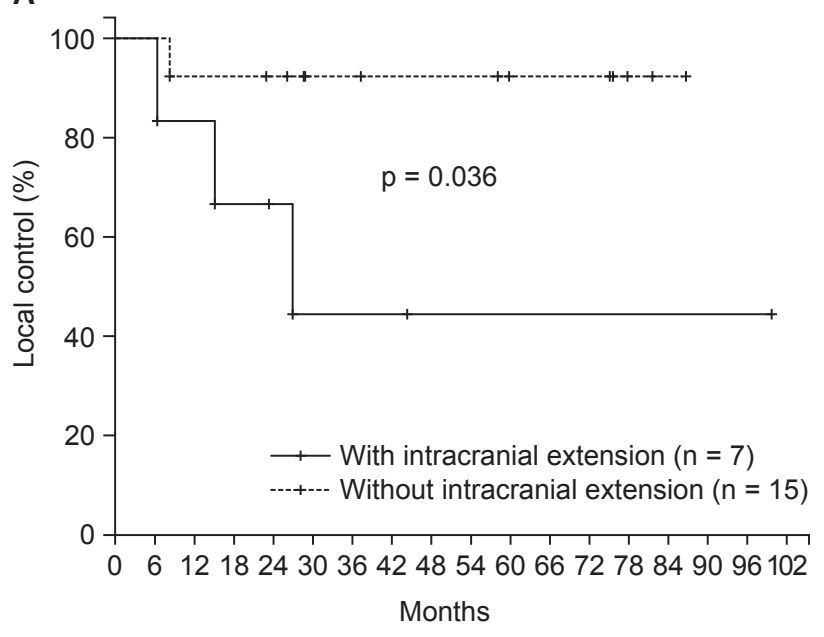

B

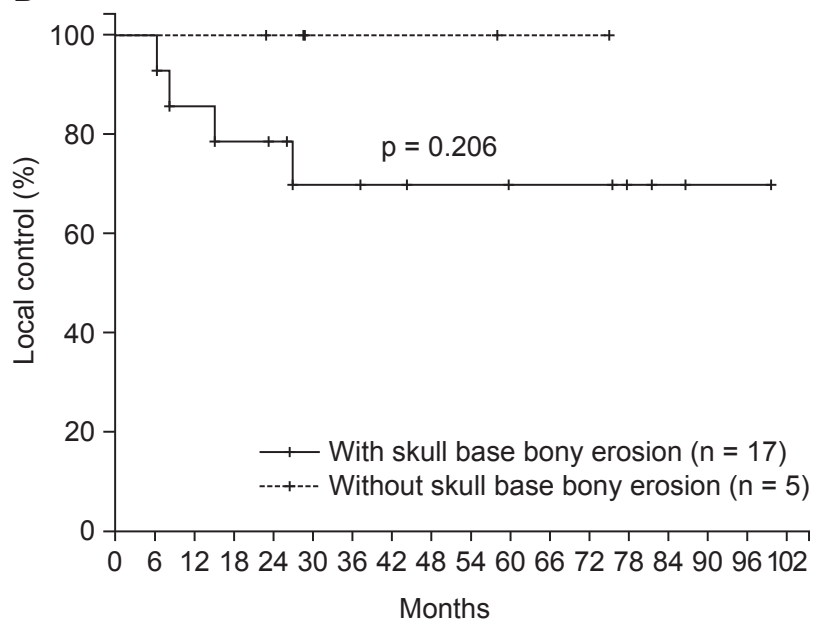

Fig. 2. Local control according to (A) intracranial extension and (B) skull base bony erosion.

in patients without distant metastases and $0 \%$ in patients with distant metastases $(p<0.001)$. The disease states at last follow-up were as follows: 6 patients (27.3\%) were alive without evidence of disease; 2 patients (9.1\%) were alive with disease; and 14 patients (63.6\%) were deceased. Among these 14 deaths, 10 patients died because of the aggravation of PM-RMS and 2 patients died because of treatment related complications (deep neck infection and thrombocytopenial nasal bleeding). Causes of death of the remaining 2 patients were difficult to assess.

Surgical resection did not affect the PFS ( $p=0.692)$ or OS ( $p$ $=0.593)$.

\section{Prognostic factors for local control}

Table 2 shows the prognostic factors for the LC. ICE was
Table 2. Prognostic factor for local control in parameningeal rhabdomyosarcoma

\begin{tabular}{|c|c|c|c|}
\hline \multirow{2}{*}{ Variable } & \multirow{2}{*}{ No. (\%) } & \multicolumn{2}{|c|}{ Local control } \\
\hline & & $3-y r(\%)$ & $p$-value \\
\hline Age (yr) & & & 0.786 \\
\hline$<10$ & $5(22.7)$ & 75.0 & \\
\hline$\geq 10$ & 17 (77.3) & 78.0 & \\
\hline Histology & & & 0.071 \\
\hline Alveolar & $9(40.9)$ & 62.3 & \\
\hline Others & $13(59.1)$ & 100 & \\
\hline Tumor site & & & 0.670 \\
\hline $\begin{array}{l}\text { Nasopharynx and } \\
\text { nasal cavity }\end{array}$ & $13(59.1)$ & 80.8 & \\
\hline $\begin{array}{l}\text { Paranasal sinus, } \\
\text { infratemporal fossa, } \\
\text { and PPF }\end{array}$ & $9(40.9)$ & 75.0 & \\
\hline ICE & & & 0.036 \\
\hline Yes & $7(31.8)$ & 44.4 & \\
\hline No & 15 (68.2) & 92.3 & \\
\hline SBBE & & & 0.206 \\
\hline Yes & $17(77.3)$ & 69.8 & \\
\hline No & $5(22.7)$ & 100 & \\
\hline CNP & & & 0.620 \\
\hline Yes & $1(4.5)$ & 100 & \\
\hline No & $21(95.5)$ & 76.4 & \\
\hline Tumor size $(\mathrm{cm})$ & & & 0.455 \\
\hline$<5$ & $2(9.1)$ & 74.9 & \\
\hline$\geq 5$ & 20 (90.9) & 100 & \\
\hline Disease extent & & & 0.400 \\
\hline Mo & 17 (77.3) & 80.2 & \\
\hline M1 & $5(22.7)$ & 66.7 & \\
\hline Surgery & & & 0.280 \\
\hline Yes & $4(18.2)$ & 100 & \\
\hline No & 18 (81.8) & 72.0 & \\
\hline RT dose (Gy) & & & 0.501 \\
\hline$<50$ & $4(18.2)$ & 66.7 & \\
\hline$\geq 50$ & 18 (81.8) & 79.5 & \\
\hline Timing of RT (wk) & & & 0.200 \\
\hline 4 (ref. 0-6) & $5(22.7)$ & 66.7 & \\
\hline 13 (ref. 7-15) & $10(45.5)$ & 90.0 & \\
\hline 20 (ref. 16-22) & $2(9.1)$ & 100 & \\
\hline Delayed (ref. >22) & $5(22.7)$ & 50.0 & \\
\hline RT modality & & & 0.229 \\
\hline 3D-CRT & $17(77.3)$ & 57.1 & \\
\hline IMRT & $5(22.7)$ & 100 & \\
\hline
\end{tabular}

PPF, pterygopalatine fossa; ICE, intracranial extension; SBBE, skull base bony invasion; CNP, cranial nerve palsy; RT, radiotherapy; 3D-CRT, three-dimensional conformal radiotherapy; IMRT, intensity-modulated radiotherapy.

related to lower local control rate $(p=0.036)$ (Fig. $2 A)$ and alveolar histologic subtype tended to be related to lower LC 
Table 3. Clinical risk factors and treatment outcomes according to the timing of radiotherapy

\begin{tabular}{|c|c|c|c|c|}
\hline & & Timing & apy (wk) & \\
\hline & $\begin{array}{c}4 \\
\text { (ref. 0-6) } \\
(n=5)\end{array}$ & $\begin{array}{c}13 \\
\text { (ref. } 7-15) \\
(n=10)\end{array}$ & $\begin{array}{c}20 \\
\text { (ref. 16-22) } \\
(\mathrm{n}=2)\end{array}$ & $\begin{array}{c}\text { Delayed } \\
(\text { ref. }>22) \\
(n=5)\end{array}$ \\
\hline Risk factor & & & & \\
\hline ICE & $4(80)$ & $2(20)$ & $0(0)$ & $1(20)$ \\
\hline SBBE & $4(80)$ & $7(70)$ & $2(100)$ & $4(80)$ \\
\hline Disease extent & & & & \\
\hline Metastasis & $1(20)$ & $1(10)$ & $0(0)$ & $3(60)$ \\
\hline Operation & & & & \\
\hline $\begin{array}{l}\text { Surgery } \\
\text { RT dose (Gy) }\end{array}$ & $1(20)$ & $3(30)$ & 0 (0) & $0(0)$ \\
\hline$\geq 50$ & $4(80)$ & $8(80)$ & $2(100)$ & $4(80)$ \\
\hline Treatment outcome & & & & \\
\hline Local failure & $1(20)$ & $1(10)$ & $0(0)$ & $2(40)$ \\
\hline Overall progression & $2(40)$ & $4(40)$ & $0(0)$ & $4(80)$ \\
\hline
\end{tabular}

Values are presented as number (\%).

ICE, intracranial extension; SBBE, skull base bony erosion.

$(p=0.071)$ (Table 2). However, the SBBE did not significantly compromise the $L C(p=0.206)$ (Fig. 2B).

\section{Radiation dose}

Patients were divided into two groups and evaluated according to the radiation dose; the first group $(n=18)$ was composed of patients treated with an equal and greater dose of the standard guideline ( $\geq 50 \mathrm{~Gy})$. The second group $(n=4)$ was composed of patients who were treated with a reduced dose of radiation ( $<50 \mathrm{~Gy})$. Half of the patients $(n=2)$, who were treated with reduced dose radiation $(<50 \mathrm{~Gy})$, were patients under the age of 10 years. The patients with ICE did not receive a higher dose of RT (ICE 5/7 vs. non-ICE 13/15, >50 Gy) than patients without ICE. The RT dose did not significantly compromise the $L C$ rate $(p=0.501)$ (Table 2$)$. A reduced radiation dose $(<50 \mathrm{~Gy})$ also did not affect the survival outcome (PFS, $p=0.068$ and $0 S, p=0.394$, respectively).

\section{Radiotherapy timing}

Patients were divided according to the timing of the RT administration and evaluated at the following reference points: 4 weeks, 13 weeks, and 20 weeks from the time of diagnosis. Table 3 shows the occurrence of local failure and overall progression with regard to RT timing.

Causes of delay in administering RT ( $>22$ weeks) varied. Among 5 patients who received delayed RT, 2 patients experienced complications (cardiomyositis/neutropenia) after chemotherapy. Two patients were transferred to our hospital after receiving several cycles of chemotherapy in other hospitals. The other patient was too young (3 years old) at the time of initial diagnosis; therefore, chemotherapy was administered preferentially and RT was delayed for 1 year.

Patients in the delayed RT group $(n=5)$ did not undergo surgery before RT. Four patients had stage III disease and 1 patient had stage IV disease. Even though a large portion of patients who delayed RT had metastases at the time of the initial diagnosis (60.0\%) (Table 3), a delayed administration (after 22 weeks from diagnosis) of the RT was related to higher rate of local failure (40.0\%).

Among the 4 patients who underwent surgery, no patients experienced local failure and only 1 patient had ICE at diagnosis. A delay in administering RT ( $>22$ weeks) did not occur as a consequence of performing surgery. All patients who underwent surgery before RT received RT within 15 weeks (Table 3).

\section{Discussion and Conclusion}

The parameningeal site is considered to be an adverse prognostic factor in RMS [5]. However, the current study showed a substantial level of LC (3-year, 77.7\%) in the PMRMS patients. All the patients in this study received RT as an initial treatment. In this regard, the administration of RT as an initial treatment is important to achieve a higher LC in PMRMS. However, this higher LC was not extended to OS gain (3year, 51.4\%). The most common cause of treatment failure 
was distant metastasis. Moreover, all patients, who developed distant failure during the follow-up periods, eventually died.

In a recent study, Merks et al. [5] reported that age, meningeal invasion (cranial neuropathy \pm SBBE \pm ICE), tumor site, and tumor size were significant prognostic factors for PM-RMS. This study also showed that ICE was a significant prognostic factor for LC. However, age, tumor size, and tumor site did not significantly influence the treatment outcome. This discrepancy might be related to the smaller sample size of the current study.

An adequate application of RT is crucial in the PM-MRS. The RT timing and dose are important factors with regard to the effectiveness of the RT [10-12].

The timing of RT tended to be determined by the risk of intracranial (meningeal) involvement. However, it could be related to other factors, such as a young age, use of surgery or treatment toxicity [5]. The recommended timing of $\mathrm{RT}$ is variable according to protocols $[2,7,8,14]$. In general, it is recommended that patients with risk factors for progression receive RT at an early stage. However, in IRS-VI, the recommended RT timing was related to the degree of risk as follows: patients with low risk, 13 weeks; intermediate risk, 4 weeks; and high risk, 20 weeks. In the current study, a delayed RT (>22 weeks) was related to poor local control (40.0\% of local failure) (Table 3). Moreover, the poor outcome of the delayed RT group was not clearly related with the burden of pre-existing risk factors, such as ICE and SBBE (Table 3). Surgical resection was not associated with delayed RT (Table 3) or decreased local control ( $p=0.706)$. The small number of patients who were treated with surgery may be the reason for these statistically nonsignificant results. Delayed RT was related to prolonged chemotherapy administration or chemotherapy complications. The rarity of this disease might also contribute to the heterogeneity of tumors and treatment characteristics. Physicians with limited experience of this disease might have difficulty in administering prompt or appropriate treatment after diagnosis. The poor prognosis might be related to treatment delay, which resulted in a decrease in the RT effectiveness. In this regard, the administration of the RT in time is a desirable goal to achieve a better response from RT.

The radiation dose did not affect LC in this study (Table 3). This result supported the results of Puri et al. [11] Especially in young patients, a dose reduction can be considered as a treatment option to reduce radiation-induced toxicities, such as growth hormone deficiency, or growth delay, or bony deformity [15]. In regard to the reduction of the RT-induced late toxicity, the use of proton therapy or the shrinking field RT can also be considered as a treatment option [16].

This study showed a substantial rate of LC (5-year LC, 77.7\%) in the PM-RMS. The modern RT techniques improve the target dose coverage (i.e., three-dimensional conformal RT [17] or intensity modulated RT [18]). The current risk-based approach [9] and tailored multimodal treatment [19] might also contribute to the improvement in treatment outcome.

The current study had several limitations. The initial response to chemotherapy is also known to have prognostic significance [13]. Nevertheless, an early response evaluation was impossible due to a lack of imaging studies after initial chemotherapy. In addition, this study did not deal with treatment-induced toxicity, which might compromise the survival rate. Also, it was difficult to estimate the effect on local control of high dose RT in patients with meningeal involvement. To clarify the effect of the RT, a prospective long-term follow-up study is warranted. Finally, because of the rarity of PM-RMS there were a relatively small number of patients. Our data is not sufficient to evaluate a real effect of RT because of small sample size. Especially, the number of patients who treated with reduced dose irradiation was only 4. Therefore, it is difficult to conclude that dose of RT did not influence the treatment outcome. Also, the results that tumor size was not related to prognosis might be affected by the small sample size of this study. The statistical power of this study tended to be lower than that of other largescale studies. Nevertheless, this study's findings could provide important clinical information about Korean PM-RMS patients. A well-designed, multi-institutional study is warranted to evaluate this rare disease.

In conclusion, RT can allow for a high rate of $\mathrm{LC}$ for the PM-RMS patients. An adequate use of RT is an essential part of treatment for the PM-RMS. However, the benefit of LC did not extend to survival outcome because of the high rate of systemic failure. Therefore, further studies should be attempted to achieve better treatment outcomes of PM-RMS. A more effective treatment is still warranted to improve the clinical outcome of PM-RMS.

\section{Conflict of Interest}

No potential conflict of interest relevant to this article was reported.

\section{References}

1. Wharam MD Jr. Rhabdomyosarcoma of parameningeal sites. Semin Radiat Oncol 1997;7:212-6. 


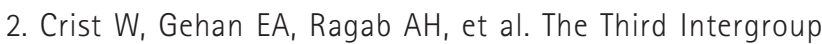
Rhabdomyosarcoma Study. J Clin Oncol 1995;13:610-30.

3. Mazzoleni S, Bisogno G, Garaventa $A$, et al. Outcomes and prognostic factors after recurrence in children and adolescents with nonmetastatic rhabdomyosarcoma. Cancer 2005;104:183-90.

4. Yang JC, Wexler LH, Meyers PA, Wolden SL. Parameningeal rhabdomyosarcoma: outcomes and opportunities. Int J Radiat Oncol Biol Phys 2013;85:e61-6.

5. Merks JH, De Salvo GL, Bergeron C, et al. Parameningeal rhabdomyosarcoma in pediatric age: results of a pooled analysis from North American and European cooperative groups. Ann Oncol 2014;25:231-6.

6. Turner JH, Richmon JD. Head and neck rhabdomyosarcoma: a critical analysis of population-based incidence and survival data. Otolaryngol Head Neck Surg 2011;145:967-73.

7. Spalding AC, Hawkins DS, Donaldson SS, et al. The effect of radiation timing on patients with high-risk features of parameningeal rhabdomyosarcoma: an analysis of IRS-IV and D9803. Int J Radiat Oncol Biol Phys 2013;87:512-6.

8. Douglas JG, Arndt CA, Hawkins DS. Delayed radiotherapy following dose intensive chemotherapy for parameningeal rhabdomyosarcoma (PM-RMS) of childhood. Eur J Cancer 2007;43:1045-50.

9. Raney RB, Maurer HM, Anderson JR, et al. The Intergroup Rhabdomyosarcoma Study Group (IRSG): major lessons from the IRS-I through IRS-IV Studies as background for the current IRS-V treatment protocols. Sarcoma 2001;5:9-15.

10. Michalski JM, Meza J, Breneman JC, et al. Influence of radiation therapy parameters on outcome in children treated with radiation therapy for localized parameningeal rhabdomyosarcoma in Intergroup Rhabdomyosarcoma Study Group trials II through IV. Int J Radiat Oncol Biol Phys 2004;59:1027-38.

11. Puri DR, Wexler LH, Meyers PA, La Quaglia MP, Healey JH, Wolden SL. The challenging role of radiation therapy for very young children with rhabdomyosarcoma. Int J Radiat Oncol Biol Phys 2006;65:1177-84.

12. Breneman J, Meza J, Donaldson SS, et al. Local control with reduced-dose radiotherapy for low-risk rhabdomyosarcoma: a report from the Children's Oncology Group D9602 study. Int J Radiat Oncol Biol Phys 2012;83:720-6.

13. Park JA, Kim EK, Kang HJ, Shin HY, Kim IH, Ahn HS. Initial response to treatment was highly associated with the prognosis of childhood rhabdomyosarcoma: a retrospective analysis of a single center experience in Korea. Cancer Res Treat 2008;40:111-5.

14. Crist WM, Anderson JR, Meza JL, et al. Intergroup rhabdomyosarcoma study-IV: results for patients with nonmetastatic disease. J Clin Oncol 2001;19:3091-102.

15. Raney RB, Asmar L, Vassilopoulou-Sellin R, et al. Late complications of therapy in 213 children with localized nonorbital soft-tissue sarcoma of the head and neck: a descriptive report from the Intergroup Rhabdomyosarcoma Studies (IRS)-II and -III. IRS Group of the Children's Cancer Group and the Pediatric Oncology Group. Med Pediatr Oncol 1999:33:362-71.

16. Childs SK, Kozak KR, Friedmann AM, et al. Proton radiotherapy for parameningeal rhabdomyosarcoma: clinical outcomes and late effects. Int J Radiat Oncol Biol Phys 2012;82:635-42.

17. Wolden SL, La TH, LaQuaglia MP, Meyers PA, Kraus DH, Wexler LH. Long-term results of three-dimensional conformal radiation therapy for patients with rhabdomyosarcoma. Cancer 2003;97:179-85.

18. Wolden SL, Wexler LH, Kraus DH, Laquaglia MP, Lis $E_{1}$ Meyers PA. Intensity-modulated radiotherapy for headand-neck rhabdomyosarcoma. Int J Radiat Oncol Biol Phys 2005;61:1432-8.

19. Hawkins DS, Anderson JR, Paidas CN, et al. Improved outcome for patients with middle ear rhabdomyosarcoma: a children's oncology group study. J Clin Oncol 2001;19:3073-9. 